Arezki Touzaline (Bab Ezzouar)

\title{
A QUASISTATIC UNILATERAL AND FRICTIONAL CONTACT PROBLEM WITH ADHESION FOR ELASTIC MATERIALS
}

Abstract. We consider a quasistatic contact problem between a linear elastic body and a foundation. The contact is modelled with the Signorini condition and the associated non-local Coulomb friction law in which the adhesion of the contact surfaces is taken into account. The evolution of the bonding field is described by a first order differential equation. We derive a variational formulation of the mechanical problem and prove existence of a weak solution if the friction coefficient is sufficiently small. The proofs employ a time-discretization method, compactness and lower semicontinuity arguments, differential equations and the Banach fixed point theorem.

1. Introduction. Contact problems involving deformable bodies are quite frequent in industry as well as in daily life and play an important role in structural and mechanical systems. Because of the importance of such processes a considerable effort has been put into their modelling and numerical simulations. A first study of frictional contact problems within the framework of variational inequalities was made in [8]. The mathematical, mechanical and numerical state of the art can be found in [15]. In this paper we study a quasistatic unilateral contact problem with a non-local Coulomb friction law and adhesion between a linear elastic body and an obstacle, the so-called foundation. Models for dynamic or quasistatic processes of frictionless adhesive contact between a deformable body and a foundation have been studied in $[3,4,11,20]$. As in $[10,11]$ we use the bonding field $\beta$ as an additional state variable, defined on the contact surface of the boundary.

2000 Mathematics Subject Classification: 74M10, 74M15, 47J20, $49 \mathrm{~J} 40$.

Key words and phrases: elastic materials, adhesion, quasistatic, time-discretization, friction, fixed point, weak solution. 
The variable is restricted to values $0 \leq \beta \leq 1$, when $\beta=0$ all the bonds are severed and there are no active bonds; when $\beta=1$ all the bonds are active; when $0<\beta<1$ it measures the fraction of active bonds and partial adhesion takes place. We refer the reader to the extensive bibliography on the subject in $[14-17,19]$. In [2] a model of a contact problem with adhesion and friction was studied in which $\beta$ represents a continuous transition between total adhesive and pure frictional states. As in [5], in this work by applying a time-discretization method, we prove that the time-discretized problem has a unique solution if the friction coefficient is sufficiently small. We finally obtain the existence of a weak solution by passing to the limit with respect to time.

The paper is structured as follows. In Section 2 we present some notations and give a variational formulation. In Section 3 we study a time-discretized problem which admits a unique solution if the friction coefficient is small enough (Proposition 3.2). In Section 4 we prove our existence theorem.

2. Problem statement and variational formulation. Let $\Omega \subset \mathbb{R}^{d}$ $(d=2,3)$ be the domain initially occupied by an elastic body. Here $\Omega$ is supposed to be open, bounded, with a sufficiently regular boundary $\Gamma$, partitioned into three parts, $\Gamma=\bar{\Gamma}_{1} \cup \bar{\Gamma}_{2} \cup \bar{\Gamma}_{3}$, where $\Gamma_{1}, \Gamma_{2}, \Gamma_{3}$ are disjoint open sets and meas $\Gamma_{1}>0$. The body is acted upon by a volume force of density $\varphi_{1}$ on $\Omega$ and a surface traction of density $\varphi_{2}$ on $\Gamma_{2}$. On $\Gamma_{3}$ the body is in adhesive frictional contact with a foundation.

Thus, the classical formulation of the mechanical problem is as follows.

Problem $P_{1}$. Find a displacement field $u: \Omega \times[0, T] \rightarrow \mathbb{R}^{d}$ and a bonding field $\beta: \Gamma_{3} \times[0, T] \rightarrow[0,1]$ such that

$$
\begin{gathered}
\begin{array}{cc}
\operatorname{div} \sigma+\varphi_{1}=0 & \text { in } \Omega \times(0, T), \\
\left(\sigma_{i j}\right)=\left(a_{i j k l} \varepsilon_{k l}(u)\right) & \text { in } \Omega \times(0, T), \\
u=0 & \text { on } \Gamma_{1} \times(0, T), \\
\sigma \nu=\varphi_{2} & \text { on } \Gamma_{2} \times(0, T), \\
u_{\nu} \leq 0, & \sigma_{\nu}-c_{\nu} \beta^{2} R_{\nu}\left(u_{\nu}\right) \leq 0, \\
\sigma_{\nu}-c_{\nu} \beta^{2} R_{\nu}\left(u_{\nu}\right) u_{\nu}=0 & \text { on } \Gamma_{3} \times(0, T),
\end{array} \\
\left\{\begin{array}{rr}
\left|\sigma_{\tau}+c_{\tau} \beta^{2} R_{\tau}\left(u_{\tau}\right)\right| \leq \mu\left|R^{*}\left(\sigma_{\nu}(u)\right)\right| & \text { on } \Gamma_{3} \times(0, T), \\
\left|\sigma_{\tau}+c_{\tau} \beta^{2} R_{\tau}\left(u_{\tau}\right)\right|<\mu\left|R^{*}\left(\sigma_{\nu}(u)\right)\right| \Rightarrow \dot{u}_{\tau}=0, & \\
\left|\sigma_{\tau}+c_{\tau} \beta^{2} R_{\tau}\left(u_{\tau}\right)\right|=\mu\left|R^{*}\left(\sigma_{\nu}(u)\right)\right| & \Rightarrow \exists \lambda \geq 0: \dot{u}_{\tau}=-\lambda\left(\sigma_{\tau}+c_{\tau} \beta^{2} R_{\tau}\left(u_{\tau}\right)\right),
\end{array}\right.
\end{gathered}
$$




$$
\begin{array}{cl}
\dot{\beta}=-\left[\beta\left(c_{\nu}\left(R_{\nu}\left(u_{\nu}\right)\right)^{2}+c_{\tau}\left(\left|R_{\tau}\left(u_{\tau}\right)\right|\right)^{2}\right)-\varepsilon_{a}\right]_{+} & \text {on } \Gamma_{3} \times(0, T), \\
u(0)=u_{0} & \text { in } \Omega, \\
\beta(0)=\beta_{0} & \text { on } \Gamma_{3} .
\end{array}
$$

Equation (2.1) is the equilibrium equation. Equation (2.2) represents the elastic constitutive law of the material with $\left(\sigma_{i j}\right)$ denoting the stress tensor and $\left(\varepsilon_{k l}(u)\right)$ the small strain tensor; (2.3) and (2.4) are the displacement and traction boundary conditions, respectively, in which $\nu$ denotes the unit outward normal vector on $\Gamma$ and $\sigma \nu$ is the Cauchy stress vector. Conditions (2.5) represent the unilateral contact with adhesion. Conditions (2.6) represent a non-local frictional contact in which the adhesion is taken into account and $\dot{u}_{\tau}$ is the tangential velocity on the boundary. The tangential shear cannot exceed the maximal frictional resistance $\mu\left|R^{*}\left(\sigma_{\nu}(u)\right)\right|$. If the strict inequality is satisfied, the surface adheres to the foundation and is in the so-called stick state, and when equality is satisfied there is relative sliding, the so-called slip state. Here $R^{*}$ is a compact regularization operator (see [7]) and the parameters $c_{\nu}, c_{\tau}$ and $\varepsilon_{a}$ are given adhesion coefficients which may depend on $x \in \Gamma_{3}$. As in [18], $R_{\nu}, R_{\tau}$ are truncation operators defined by

$$
R_{\nu}(s)=\left\{\begin{array}{ll}
L & \text { if } s<-L, \\
-s & \text { if }-L \leq s \leq 0, \\
0 & \text { if } s>0,
\end{array} \quad R_{\tau}(v)= \begin{cases}v & \text { if }|v| \leq L, \\
L v /|v| & \text { if }|v|>L,\end{cases}\right.
$$

where $L>0$ is a characteristic length of the bonds. Equation (2.7), where $[s]_{+}=\max (s, 0)$ for $s \in \mathbb{R}$, describes the evolution of the bonding field and was already used in [18]. Since $\dot{\beta} \leq 0$ on $\Gamma_{3} \times(0, T)$, once debonding occurs, bonding cannot be reestablished. Also we wish to make it clear that from [13] it follows that the model does not allow for a complete debonding field in finite time. Finally, (2.8) and (2.9) are the the initial conditions in which $u_{0}$ and $\beta_{0}$ denote respectively the initial displacement field and the initial bonding field. In (2.7) the dot above a variable represents its derivative with respect to time.

We recall that the inner products and the corresponding norms on $\mathbb{R}^{d}$ and $S_{d}$ are given by

$$
\begin{array}{lll}
u . v=u_{i} v_{i}, & |v|=(v . v)^{1 / 2} & \forall u, v \in \mathbb{R}^{d}, \\
\sigma . \tau=\sigma_{i j} \tau_{i j}, & |\tau|=(\tau . \tau)^{1 / 2} & \forall \sigma, \tau \in S_{d},
\end{array}
$$

where $S_{d}$ is the space of second order symmetric tensors on $\mathbb{R}^{d}(d=2,3)$. Here and below, the indices $i$ and $j$ run between 1 and $d$ and the summation convention over repeated indices is adopted. 
Now, to proceed with the variational formulation, we need the following function spaces:

$$
\begin{aligned}
& H=\left(L^{2}(\Omega)\right)^{d}, \quad H_{1}=\left(H^{1}(\Omega)\right)^{d}, \\
& Q=\left\{\tau=\left(\tau_{i j}\right): \tau_{i j}=\tau_{j i} \in L^{2}(\Omega)\right\}, \quad Q_{1}=\{\sigma \in Q: \operatorname{div} \sigma \in H\} .
\end{aligned}
$$

Note that $H$ and $Q$ are real Hilbert spaces endowed with the respective canonical inner products

$$
\langle u, v\rangle_{H}=\int_{\Omega} u_{i} v_{i} d x, \quad\langle\sigma, \tau\rangle_{Q}=\int_{\Omega} \sigma_{i j} \tau_{i j} d x .
$$

The small strain tensor is

$$
\varepsilon(u)=\left(\varepsilon_{i j}(u)\right)=\frac{1}{2}\left(u_{i, j}+u_{j, i}\right), \quad i, j=\{1, \ldots, d\} ;
$$

$\operatorname{div} \sigma=\left(\sigma_{i j, j}\right)$ is the divergence of $\sigma$. For every element $v \in H_{1}$ we denote by $v_{\nu}$ and $v_{\tau}$ the normal and the tangential components of $v$ on the boundary $\Gamma$, given by

$$
v_{\nu}=v . \nu, \quad v_{\tau}=v-v_{\nu} \nu
$$

Similarly, for a regular tensor field $\sigma \in Q_{1}$, we define its normal and tangential components by

$$
\sigma_{\nu}=(\sigma \nu) . \nu, \quad \sigma_{\tau}=\sigma \nu-\sigma_{\nu} \nu,
$$

and we recall that the following Green formula holds:

$$
\langle\sigma, \varepsilon(v)\rangle_{Q}+\langle\operatorname{div} \sigma, v\rangle_{H}=\int_{\Gamma} \sigma \nu \cdot v d a \quad \forall v \in H_{1},
$$

where $d a$ is the surface measure element. Let $V$ be the closed subspace of $H_{1}$ defined by

$$
V=\left\{v \in H_{1}: v=0 \text { on } \Gamma_{1}\right\},
$$

and let $K$ be the set of admissible displacements of $V$ defined by

$$
K=\left\{v \in V: v_{\nu} \leq 0 \text { a.e. on } \Gamma_{3}\right\} .
$$

Since meas $\Gamma_{1}>0$, the following Korn inequality holds [8]:

$$
\|\varepsilon(v)\|_{Q} \geq c_{\Omega}\|v\|_{H_{1}} \quad \forall v \in V
$$

where the constant $c_{\Omega}>0$ depends only on $\Omega$ and $\Gamma_{1}$. We equip $V$ with the inner product

$$
(u, v)_{V}=\langle\varepsilon(u), \varepsilon(v)\rangle_{Q}
$$

and $\|\cdot\|_{V}$ is the associated norm. It follows from Korn's inequality (2.10) that the norms $\|\cdot\|_{H_{1}}$ and $\|\cdot\|_{V}$ are equivalent on $V$. Then $\left(V,\|\cdot\|_{V}\right)$ is a real Hilbert space. Moreover, by Sobolev's trace theorem, there exists $d_{\Omega}>0$ which depends only on the domain $\Omega, \Gamma_{1}$ and $\Gamma_{3}$ such that

$$
\|v\|_{\left(L^{2}\left(\Gamma_{3}\right)\right)^{d}} \leq d_{\Omega}\|v\|_{V} \quad \forall v \in V .
$$


For $p \in[1, \infty]$, we use the standard norm of $L^{p}(0, T ; V)$. We also use the Sobolev space $W^{1, \infty}(0, T ; V)$ equipped with the norm

$$
\|v\|_{W^{1, \infty}(0, T ; V)}=\|v\|_{L^{\infty}(0, T ; V)}+\|\dot{v}\|_{L^{\infty}(0, T ; V)} .
$$

For every real Banach space $\left(X,\|\cdot\|_{X}\right)$ and $T>0$ we use the notation $C([0, T] ; X)$ for the space of continuous functions from $[0, T]$ to $X$; recall that $C([0, T] ; X)$ is a real Banach space with the norm

$$
\|x\|_{C([0, T] ; X)}=\max _{t \in[0, T]}\|x(t)\|_{X} .
$$

We suppose that the body forces and surface tractions have the regularity

$$
\varphi_{1} \in W^{1, \infty}(0, T ; H), \quad \varphi_{2} \in W^{1, \infty}\left(0, T ;\left(L^{2}\left(\Gamma_{2}\right)\right)^{d}\right)
$$

and we denote by $f(t)$ the element of $V$ defined by

$$
(f(t), v)_{V}=\int_{\Omega} \varphi_{1}(t) \cdot v d x+\int_{\Gamma_{2}} \varphi_{2}(t) \cdot v d a \quad \forall v \in V, t \in[0, T] .
$$

Using (2.12) and (2.13) yields

$$
f \in W^{1, \infty}(0, T ; V) .
$$

Let

$$
H^{1 / 2}\left(\Gamma_{3}\right)=\left\{\left.w\right|_{\Gamma_{3}}: w \in H^{1 / 2}(\Gamma), w=0 \text { on } \Gamma_{1}\right\},
$$

equipped with the norm of $H^{1 / 2}(\Gamma)$. Let $\langle\cdot, \cdot\rangle_{\Gamma_{3}}$ denote the duality pairing on $H^{1 / 2}\left(\Gamma_{3}\right) \times H^{-1 / 2}\left(\Gamma_{3}\right)$.

Before we start with the variational formulation of Problem $P_{1}$ let us state in which sense the duality pairing $\langle\cdot, \cdot\rangle$ is taken. For $\sigma \in Q_{1}$, if $\sigma \nu \in$ $\left(L^{2}\left(\Gamma_{2}\right)\right)^{d}$ in the sense of distributions, i.e. there exists $s \in\left(L^{2}\left(\Gamma_{2}\right)\right)^{d}$ such that

$$
\langle\sigma \nu, \varphi\rangle_{\mathbb{H}^{-1 / 2}(\Gamma) \times \mathbb{H}^{1 / 2}(\Gamma)}=\int_{\Gamma_{2}} s . \varphi d a \quad \forall \varphi \in\left(C_{0}^{\infty}\left(\Gamma_{2}\right)\right)^{d},
$$

where $\mathbb{H}^{1 / 2}(\Gamma)=\left(H^{1 / 2}(\Gamma)\right)^{d}$ and $\mathbb{H}^{-1 / 2}(\Gamma)=\left(H^{-1 / 2}(\Gamma)\right)^{d}$, we define the normal stress $\sigma_{\nu}$ on $\Gamma_{3}$ as follows:

$$
\left\{\begin{array}{l}
\forall w \in H^{1 / 2}\left(\Gamma_{3}\right): \\
\left\langle\sigma_{\nu}, w\right\rangle_{\Gamma_{3}}=\langle\sigma, \varepsilon(v)\rangle_{Q}+\langle\operatorname{div} \sigma, v\rangle_{H}-\int_{\Gamma_{2}} s . v d a \\
\forall v \in V \text { with } v_{\nu}=w \text { and } v_{\tau}=0 \text { on } \Gamma_{3} .
\end{array}\right.
$$

We assume that $R^{*}: H^{-1 / 2}\left(\Gamma_{3}\right) \rightarrow L^{2}\left(\Gamma_{3}\right)$ is a compact linear mapping. Now, in the study of the mechanical problem $P_{1}$ we suppose that $a_{i j k l} \in$ $L^{\infty}(\Omega), 1 \leq i, j, k, l \leq d$, with the usual conditions of symmetry and ellip- 
ticity, that is,

- $a_{i j k l}=a_{j i k l}=a_{k l i j}, 1 \leq i, j, k, l \leq d$,

- $\exists \alpha>0 \forall \eta=\left(\eta_{i j}\right) \in \mathbb{R}^{d \times d}$ with $\eta_{i j}=\eta_{j i}, 1 \leq i, j, k, l \leq d$,

$$
a_{i j k l} \eta_{i j} \eta_{i j} \geq \alpha|\eta|^{2} \text {. }
$$

We define the bilinear form $a(\cdot, \cdot)$ on $V \times V$ by

$$
a(u, v)=\int_{\Omega} a_{i j k l} \varepsilon_{i j}(u) \varepsilon_{k l}(v) d x .
$$

It follows from Korn's inequality that $a(\cdot, \cdot)$ is continuous and coercive, that is,

$$
\left\{\begin{array}{c}
\text { (a) there exists } M>0 \text { such that } \\
\qquad|a(u, v)| \leq M\|u\|_{V} \|_{v} \quad \forall u, v \in V, \\
\text { (b) there exists } m>0 \text { such that } \\
|a(v, v)| \geq m\|v\|_{V}^{2} \quad \forall v \in V .
\end{array}\right.
$$

Also as in [6] we define the functional $j: V \times V \rightarrow \mathbb{R}$ by

$$
j(u, v)=\int_{\Gamma_{3}} \mu\left|R^{*}\left(\sigma_{\nu}(P u)\right)\right|\left|v_{\tau}\right| d a \quad \forall u, v \in V,
$$

where $P$ is the projection from $V$ onto $V_{0}=\left\{v \in V: \operatorname{div} \sigma(v)+\varphi_{1}=0\right.$ in $\left.\Omega\right\}$. If $u$ is a solution of Problem $P_{2}$ stated below then $P u=u$.

As in [18], we suppose that the adhesion coefficients $c_{\nu}, c_{\tau}$ and $\varepsilon_{a}$ satisfy the conditions

$$
c_{\nu}, c_{\tau} \in L^{\infty}\left(\Gamma_{3}\right), \quad \varepsilon_{a} \in L^{\infty}\left(\Gamma_{3}\right), \quad c_{\nu}, c_{\tau}, \varepsilon_{a} \geq 0 \quad \text { a.e. on } \Gamma_{3} .
$$

The friction coefficient $\mu$ satisfies

$$
\mu \in L^{\infty}\left(\Gamma_{3}\right) \text { and } \mu \geq 0 \text { a.e. on } \Gamma_{3} \text {. }
$$

We need the following set of bonding fields:

$$
B=\left\{\beta \in L^{\infty}\left(0, T ; L^{2}\left(\Gamma_{3}\right)\right): 0 \leq \beta(t) \leq 1 \forall t \in[0, T] \text {, a.e. on } \Gamma_{3}\right\} .
$$

Also, we define the adhesion functionals $r_{\nu}: L^{\infty}\left(\Gamma_{3}\right) \times V \times V \rightarrow \mathbb{R}$ and $r_{\tau}: L^{\infty}\left(\Gamma_{3}\right) \times V \times V \rightarrow \mathbb{R}$ by

$$
\begin{array}{ll}
r_{\nu}(\beta, u, v)=-\int_{\Gamma_{3}} c_{\nu} \beta^{2} R_{\nu}\left(u_{\nu}\right) v_{\nu} d a & \forall(\beta, u, v) \in L^{\infty}\left(\Gamma_{3}\right) \times V \times V, \\
r_{\tau}(\beta, u, v)=\int_{\Gamma_{3}} c_{\tau} \beta^{2} R_{\tau}\left(u_{\tau}\right) \cdot v_{\tau} d a & \forall(\beta, u, v) \in L^{\infty}\left(\Gamma_{3}\right) \times V \times V .
\end{array}
$$

Finally, we assume that the initial displacement field $u_{0}$ belongs to $K$ and

$$
\begin{aligned}
a\left(u_{0}, v-u_{0}\right)+j\left(u_{0}, v-u_{0}\right)+r & \left(\beta_{0}, u_{0}, v-u_{0}\right) \\
& \geq\left(f(0), v-u_{0}\right)_{V} \quad \forall v \in K,
\end{aligned}
$$


where

$$
r=r_{\nu}+r_{\tau}
$$

and the initial bonding field $\beta_{0}$ satisfies

$$
\beta_{0} \in L^{2}\left(\Gamma_{3}\right), \quad 0 \leq \beta_{0} \leq 1 \quad \text { a.e. on } \Gamma_{3} .
$$

As in [5], using Green's formula, we obtain the following variational formulation for Problem $P_{1}$.

Problem $P_{2}$. Find a displacement field $u \in W^{1, \infty}(0, T ; V)$ and a bonding field $\beta \in W^{1, \infty}\left(0, T ; L^{2}\left(\Gamma_{3}\right)\right) \cap B$ such that $u(0)=u_{0}, \beta(0)=\beta_{0}$ and for almost all $t \in[0, T], u(t) \in K$ and

$$
\begin{gathered}
(2.20) \quad a(u(t), v-\dot{u}(t))+j(u(t), v)-j(u(t), \dot{u}(t)) \\
+r_{\tau}(\beta(t), u(t), v-\dot{u}(t)) \\
\geq(f(t), v-\dot{u}(t))_{V}+\left\langle\sigma_{\nu}(u(t)), v_{\nu}-\dot{u}_{\nu}(t)\right\rangle_{\Gamma_{3}} \quad \forall v \in V, \\
(2.21) \quad\left\langle\sigma_{\nu}(u(t)), z_{\nu}-u_{\nu}(t)\right\rangle_{\Gamma_{3}}+r_{\nu}(\beta(t), u(t), z-u(t)) \geq 0 \quad \forall z \in K, \\
(2.22) \quad \dot{\beta}(t)=-\left[\beta(t)\left(c_{\nu}\left(R_{\nu}\left(u_{\nu}(t)\right)\right)^{2}+c_{\tau}\left(\left|R_{\tau}\left(u_{\tau}(t)\right)\right|\right)^{2}\right)-\varepsilon_{a}\right]_{+} \text {a.e. on } \Gamma_{3},
\end{gathered}
$$

Our main result, which will be established in Section 4, is the following theorem.

Theorem 2.1. Let $T>0$ and assume (2.12) and (2.15)-(2.19). Then Problem $P_{2}$ has at least one solution if the friction coefficient is sufficiently small.

3. A time-discretized formulation. This evolution problem can be integrated in time by an implicit scheme as in [5]. We need a partition of the time interval $[0, T], 0=t_{0}<t_{1}<\cdots<t_{n}=T$, where $t_{i}=i \Delta t, 0 \leq i \leq n$, with step size $\Delta t=T / n$. We denote by $\left(u^{i}, \beta^{i}\right)$ the approximation of $(u, \beta)$ at time $t_{i}$ and $\Delta u^{i}=u^{i+1}-u^{i}, \Delta \beta^{i}=\beta^{i+1}-\beta^{i}$. For a continuous function $w(t)$ we set $w^{i}=w\left(t_{i}\right)$. Then we obtain a sequence of incremental problems $P_{n}^{i}$ defined for $\left(u^{0}, \beta^{0}\right)=\left(u_{0}, \beta_{0}\right)$ by:

Problem $P_{n}^{i}$. For $\left(u^{i}, \beta^{i}\right) \in K \times L^{\infty}\left(\Gamma_{3}\right)$, find $\left(u^{i+1}, \beta^{i+1}\right) \in K \times L^{\infty}\left(\Gamma_{3}\right)$ such that

$$
\left\{\begin{array}{r}
\begin{array}{r}
a\left(u^{i+1}, w-u^{i+1}\right)+j\left(u^{i+1}, w-u^{i}\right)-j\left(u^{i+1}, \Delta u^{i}\right) \\
\quad+r_{\tau}\left(\beta^{i+1}, u^{i+1}, w-u^{i+1}\right)
\end{array} \\
\geq\left(f^{i+1}, w-u^{i+1}\right)_{V}+\left\langle\sigma_{\nu}\left(u^{i+1}\right), w_{\nu}-u_{\nu}^{i+1}\right\rangle_{\Gamma_{3}} \quad \forall w \in V, \\
r_{\nu}\left(\beta^{i+1}, u^{i+1}, z-u^{i+1}\right)+\left\langle\sigma_{\nu}\left(u^{i+1}\right), z_{\nu}-u_{\nu}^{i+1}\right\rangle_{\Gamma_{3}} \geq 0 \quad \forall z \in K, \\
\frac{\beta^{i+1}-\beta^{i}}{\Delta t}=-\left[\beta^{i+1}\left(c_{\nu}\left(R_{\nu}\left(u_{\nu}^{i+1}\right)\right)^{2}+c_{\tau}\left(\left|R_{\tau}\left(u_{\tau}^{i+1}\right)\right|\right)^{2}\right)-\varepsilon_{a}\right]_{+} \text {a.e. on } \Gamma_{3} .
\end{array}\right.
$$


Lemma 3.1. Problem $P_{n}^{i}$ is equivalent to the following:

Problem $Q_{n}^{i}$. For $\left(u^{i}, \beta^{i}\right) \in K \times L^{\infty}\left(\Gamma_{3}\right)$, find $\left(u^{i+1}, \beta^{i+1}\right) \in K \times$ $L^{\infty}\left(\Gamma_{3}\right)$ such that

$$
\left\{\begin{array}{r}
a\left(u^{i+1}, w-u^{i+1}\right)+j\left(u^{i+1}, w-u^{i}\right)-j\left(u^{i+1}, \Delta u^{i}\right) \\
\quad+r\left(\beta^{i+1}, u^{i+1}, w-u^{i+1}\right) \geq\left(f^{i+1}, w-u^{i+1}\right)_{V} \quad \forall w \in K, \\
\frac{\beta^{i+1}-\beta^{i}}{\Delta t}=-\left[\beta^{i+1}\left(c_{\nu}\left(R_{\nu}\left(u_{\nu}^{i+1}\right)\right)^{2}+c_{\tau}\left(\left|R_{\tau}\left(u_{\tau}^{i+1}\right)\right|\right)^{2}\right)-\varepsilon_{a}\right]_{+} \\
\text {a.e. on } \Gamma_{3} .
\end{array}\right.
$$

Proof. We refer the reader to [5].

Proposition 3.2. There exists $\mu_{0}>0$ such that for $\|\mu\|_{L^{\infty}\left(\Gamma_{3}\right)}<\mu_{0}$, Problem $Q_{n}^{i}$ has a unique solution.

To show Proposition 3.2 we introduce an intermediate problem. Namely, we define the closed convex set

$$
C_{+}^{*}=\left\{g \in L^{2}\left(\Gamma_{3}\right): g \geq 0 \text { a.e. on } \Gamma_{3}\right\}
$$

and the mapping $\varphi_{g}: K \rightarrow \mathbb{R}, g \in L^{2}\left(\Gamma_{3}\right)$, given by

$$
\varphi_{g}(w)=\int_{\Gamma_{3}} \mu g\left|w_{\tau}\right| d s
$$

For $g \in C_{+}^{*}$, we introduce the following problem $Q_{n g}^{i}$ defined as the contact problem with given friction on the contact zone.

Problem $Q_{n g}^{i}$. For $\left(u^{i}, \beta^{i}\right) \in K \times L^{\infty}\left(\Gamma_{3}\right)$, find $\left(u_{g}, \beta_{g}\right) \in K \times L^{\infty}\left(\Gamma_{3}\right)$ such that

$$
\left\{\begin{array}{r}
a\left(u_{g}, w-u_{g}\right)+\varphi_{g}\left(w-u^{i}\right)-\varphi_{g}\left(u_{g}-u^{i}\right)+r\left(\beta_{g}, u_{g}, w-u_{g}\right) \\
\geq\left(f^{i+1}, w-u_{g}\right)_{V} \quad \forall w \in K, \\
\frac{\beta_{g}-\beta^{i}}{\Delta t}=-\left[\beta_{g}\left(c_{\nu}\left(R_{\nu}\left(u_{g \nu}\right)\right)^{2}+c_{\tau}\left(\left|R_{\tau}\left(u_{g \tau}\right)\right|\right)^{2}\right)-\varepsilon_{a}\right]_{+} \text {a.e. on } \Gamma_{3} .
\end{array}\right.
$$

As in [5] we have the following lemma.

Lemma 3.3. For any $g \in C_{+}^{*}$, Problem $Q_{n g}^{i}$ has a unique solution $\left(u_{g}, \beta_{g}\right)$.

To prove Lemma 3.3 we introduce the following auxiliary problem.

Problem $P_{1 \beta}$. For $u^{i} \in K, \beta \in L^{\infty}\left(\Gamma_{3}\right)$, find $u(\beta) \in K$ such that

$$
\begin{aligned}
a(u(\beta), v- & u(\beta))+\varphi_{g}\left(v-u^{i}\right)-\varphi_{g}\left(u(\beta)-u^{i}\right) \\
& +r(\beta, u(\beta), v-u(\beta)) \geq\left(f^{i+1}, v-u(\beta)\right)_{V} \quad \forall v \in K .
\end{aligned}
$$

We have the following lemma.

Lemma 3.4. Problem $P_{1 \beta}$ has a unique solution. 
Proof. Let $A: V \rightarrow V$ be the operator given by

$$
\begin{aligned}
(A u, v)_{V} & =a(u, v)+r(\beta, u, v) \\
& =a(u, v)+\int_{\Gamma_{3}}\left(-c_{\nu} \beta^{2} R_{\nu}\left(u_{\nu}\right) v_{\nu}+c_{\tau} \beta^{2} R_{\tau}\left(u_{\tau}\right) \cdot v_{\tau}\right) d a .
\end{aligned}
$$

From $(2.15)(\mathrm{a}),(2.11),(2.16)$, and the properties of the operators $R_{\nu}$ and $R_{\tau}$ (see [19]):

$$
\begin{array}{ll}
\left|R_{\nu}(a)-R_{\nu}(b)\right| \leq|a-b| & \forall a, b \in \mathbb{R}, \\
\left|R_{\tau}(a)-R_{\tau}(b)\right| \leq|a-b| & \forall a, b \in \mathbb{R}^{d},
\end{array}
$$

it follows that

$$
\begin{aligned}
& \left|(A u-A v, w)_{V}\right| \\
& \quad \leq\left[M+\left(\left\|c_{\nu}\right\|_{L^{\infty}\left(\Gamma_{3}\right)}+\left\|c_{\tau}\right\|_{L^{\infty}\left(\Gamma_{3}\right)}\right) d_{\Omega}^{2}\|\beta\|_{L^{\infty}\left(\Gamma_{3}\right)}\right]\|u-v\|_{V}\|w\|_{V} .
\end{aligned}
$$

Also, we use (2.15)(b) to see that

$$
\begin{aligned}
(A u-A v, u-v)_{V} \geq & m\|u-v\|_{V}^{2}-\int_{\Gamma_{3}} \beta^{2} c_{\nu}\left(R_{\nu}\left(u_{\nu}\right)-R_{\nu}\left(v_{\nu}\right)\right)\left(u_{\nu}-v_{\nu}\right) d a \\
& +\int_{\Gamma_{3}} \beta^{2} c_{\tau}\left(R_{\tau}\left(u_{\tau}\right)-R_{\tau}\left(v_{\tau}\right)\right) \cdot\left(u_{\tau}-v_{\tau}\right) d a .
\end{aligned}
$$

As

$$
\begin{aligned}
\left(R_{\nu}\left(u_{\nu}\right)-R_{\nu}\left(v_{\nu}\right)\right)\left(u_{\nu}-v_{\nu}\right) \leq 0 & \text { a.e. on } \Gamma_{3}, \\
\left(R_{\tau}\left(u_{\tau}\right)-R_{\tau}\left(v_{\tau}\right)\right) \cdot\left(u_{\tau}-v_{\tau}\right) \geq 0 & \text { a.e. on } \Gamma_{3},
\end{aligned}
$$

we get

$$
(A u-A v, u-v)_{V} \geq m\|u-v\|_{V}^{2},
$$

which implies that $A$ is strongly monotone. Therefore $A$ is a strongly monotone and Lipschitz continuous operator. On the other hand, $\varphi_{g}$ is a convex, proper and lower semicontinuous functional, so by a classical argument of elliptic variational inequalities [1], the problem $P_{1 \beta}$ has a unique solution $u(\beta)$.

We also consider the following problem.

Problem $P_{2 \beta}$. For $\beta^{i} \in L^{\infty}\left(\Gamma_{3}\right)$ and $u \in V$, find $\beta \in L^{\infty}\left(\Gamma_{3}\right)$ such that

$$
\frac{\beta-\beta^{i}}{\Delta t}=-\left[\beta\left(c_{\nu}\left(R_{\nu}\left(u_{\nu}\right)\right)^{2}+c_{\tau}\left(\left|R_{\tau}\left(u_{\tau}\right)\right|\right)^{2}\right)-\varepsilon_{a}\right]_{+} \quad \text { a.e. on } \Gamma_{3} \text {. }
$$

Remark 3.5. Obviously, Problem $P_{2 \beta}$ has a unique solution given by

$$
\beta=\left\{\begin{array}{l}
\left.\beta^{i} \quad \text { if } c_{\nu}\left(R_{\nu}\left(u_{\nu}\right)\right)^{2}+c_{\tau}\left(\left|R_{\tau}\left(u_{\tau}\right)\right|\right)^{2}\right) \beta^{i}-\varepsilon_{a}<0, \\
\frac{\beta^{i}+\varepsilon_{a} \Delta t}{1+\Delta t\left(c_{\nu}\left(R_{\nu}\left(u_{\nu}\right)\right)^{2}+c_{\tau}\left(\left|R_{\tau}\left(u_{\tau}\right)\right|\right)^{2}\right)} \\
\quad \text { if }\left(c_{\nu}\left(R_{\nu}\left(u_{\nu}\right)\right)^{2}+c_{\tau}\left(\left|R_{\tau}\left(u_{\beta \tau}\right)\right|\right)^{2}\right) \beta^{i}-\varepsilon_{a} \geq 0 .
\end{array}\right.
$$


To complete the proof of Lemma 3.3, let $v \in V$ and $\beta(v)$ the corresponding solution of Problem $P_{2 \beta}$. Let $u(\beta(v))$ be the corresponding solution of Problem $P_{1 \beta}$ and define the mapping $T: K \rightarrow K$ as

$$
v \mapsto T(v)=u(\beta(v)) .
$$

Take $v=u_{i}, i=1,2$. As in [5, Lemma 2.3], there exists a constant $C>0$ such that

$$
\left\|T\left(u_{1}\right)-T\left(u_{2}\right)\right\|_{V} \leq C \Delta t\left\|u_{1}-u_{2}\right\|_{V}, \quad \forall u_{1}, u_{2} \in K .
$$

Then we conclude by a contraction argument that for $\Delta t$ sufficiently small, Problem $Q_{\eta g}^{i}$ has a unique solution $\left(u_{g}, \beta_{g}\right)$.

Next, we establish Proposition 3.2. Let $g_{j} \in C_{+}^{*}, j=1,2$, and $u_{g_{j}}$ the corresponding solutions. Taking $v=u_{g_{2}}$ in the inequality of (3.2) with $g=g_{1}$, and $v=u_{g_{1}}$ in the inequality of (3.2) with $g=g_{2}$, and adding the two inequalities, we get

$$
\begin{aligned}
& a\left(u_{g_{1}}-u_{g_{2}}, u_{g_{1}}-u_{g_{2}}\right) \leq r\left(\beta_{g_{1}}, u_{g_{1}}, u_{g_{2}}-u_{g_{1}}\right)+r\left(\beta_{g_{2}}, u_{g_{2}}, u_{g_{1}}-u_{g_{2}}\right) \\
& +\varphi_{g_{1}}\left(u_{g_{2}}-u^{i}\right)-\varphi_{g_{1}}\left(u_{g_{1}}-u^{i}\right)+\varphi_{g_{2}}\left(u_{g_{1}}-u^{i}\right)-\varphi_{g_{2}}\left(u_{g_{2}}-u^{i}\right) .
\end{aligned}
$$

We have

$$
\begin{aligned}
r\left(\beta_{g_{1}}, u_{g_{1}}, u_{g_{2}}-\right. & \left.u_{g_{1}}\right)+r\left(\beta_{g_{2}}, u_{g_{2}}, u_{g_{1}}-u_{g_{2}}\right) \\
= & \int_{\Gamma_{3}}\left[c_{\tau}\left(\beta_{g_{1}}-\beta_{g_{2}}\right)\left(\beta_{g_{1}}+\beta_{g_{2}}\right) R\left(u_{g_{1 \tau}}\right)\left(u_{g_{2 \tau}}-u_{g_{1 \tau}}\right)\right] d a \\
& -\int_{\Gamma_{3}}\left[c_{\nu}\left(\beta_{g_{1}}-\beta_{g_{2}}\right)\left(\beta_{g_{1}}+\beta_{g_{2}}\right) R\left(u_{g_{1 \nu}}\right)\left(u_{g_{1 \nu}}-u_{g_{2 \nu}}\right)\right] d a \\
& +\int_{\Gamma_{3}}\left[c_{\nu} \beta_{g_{2}}^{2}\left(R\left(u_{g_{1 \nu}}\right)-R\left(u_{g_{2 \nu}}\right)\right)\left(u_{g_{1 \nu}}-u_{g_{2 \nu}}\right)\right] d a \\
& +\int_{\Gamma_{3}}\left[c_{\tau} \beta_{g_{2}}^{2}\left(R\left(u_{g_{1 \tau}}\right)-R\left(u_{g_{2 \tau}}\right)\right)\left(u_{g_{2 \tau}}-u_{g_{1 \tau}}\right)\right] d a .
\end{aligned}
$$

Using the properties (3.5) we then deduce

$$
\begin{aligned}
& a\left(u_{g_{1}}-u_{g_{2}}, u_{g_{1}}-u_{g_{2}}\right) \\
& \leq \int_{\Gamma_{3}}\left[c_{\tau}\left(\beta_{g_{1}}-\beta_{g_{2}}\right)\left(\beta_{g_{1}}+\beta_{g_{2}}\right) R\left(u_{g_{1 \tau}}\right)\left(u_{g_{2 \tau}}-u_{g_{1 \tau}}\right)\right] d a \\
& \quad-\int_{\Gamma_{3}}\left[c_{\nu}\left(\beta_{g_{1}}-\beta_{g_{2}}\right)\left(\beta_{g_{1}}+\beta_{g_{2}}\right) R\left(u_{g_{1 \nu}}\right)\left(u_{g_{1 \nu}}-u_{g_{2 \nu}}\right)\right] d a \\
& \quad+\varphi_{g_{1}}\left(u_{g_{2}}-u^{i}\right)-\varphi_{g_{1}}\left(u_{g_{1}}-u^{i}\right)+\varphi_{g_{2}}\left(u_{g_{1}}-u^{i}\right)-\varphi_{g_{2}}\left(u_{g_{2}}-u^{i}\right) .
\end{aligned}
$$

Now, from (2.11), (2.15)(b), $\left|R_{\nu}\left(u_{\nu}\right)\right| \leq L$ and $\left|R_{\tau}\left(u_{\tau}\right)\right| \leq L$, it follows that there exists a constant $C>0$ such that

$$
\left\|u_{g_{1}}-u_{g_{2}}\right\|_{V} \leq C\left(\left\|\beta_{g_{1}}-\beta_{g_{2}}\right\|_{L^{2}\left(\Gamma_{3}\right)}+\|\mu\|_{L^{\infty}\left(\Gamma_{3}\right)}\left\|g_{1}-g_{2}\right\|_{L^{2}\left(\Gamma_{3}\right)}\right) .
$$


On the other hand, using (2.11) and the properties (3.4), we deduce from the equality of (3.2) that there exists a constant $C^{\prime}>0$ such that

$$
\left\|\beta_{g_{1}}-\beta_{g_{2}}\right\|_{L^{2}\left(\Gamma_{3}\right)} \leq C^{\prime} \Delta t\left\|u_{g_{1}}-u_{g_{2}}\right\|_{V} .
$$

Then

$$
\left(1-C C^{\prime} \Delta t\right)\left\|u_{g_{1}}-u_{g_{2}}\right\|_{V} \leq C\|\mu\|_{L^{\infty}\left(\Gamma_{3}\right)}\left\|g_{1}-g_{2}\right\|_{L^{2}\left(\Gamma_{3}\right)} .
$$

Therefore if $\Delta t<1 / C C^{\prime}$, then there exists a constant $C_{1}^{\prime}>0$ such that

$$
\left\|u_{g_{1}}-u_{g_{2}}\right\|_{V} \leq C_{1}^{\prime}\|\mu\|_{L^{\infty}\left(\Gamma_{3}\right)}\left\|g_{1}-g_{2}\right\|_{L^{2}\left(\Gamma_{3}\right)} .
$$

To end the proof, define the mapping $\Phi$ as

$$
\Phi: C_{+}^{*} \rightarrow C_{+}^{*}, \quad g \mapsto \Phi(g)=\left|R^{*}\left(\sigma_{\nu}\left(u_{g}\right)\right)\right| .
$$

We have the following result.

Lemma 3.6. There exists a constant $\mu_{0}>0$ such that if

$$
\|\mu\|_{L^{\infty}\left(\Gamma_{3}\right)}<\mu_{0}
$$

then the mapping $\Phi$ admits a unique fixed point $g_{*}$ and $\left(u_{g_{*}}, \beta_{g_{*}}\right)$ is a unique solution to Problem $Q_{n}^{i}$.

Proof. Let $g_{1}, g_{2} \in C_{+}^{*}$. Then

$$
\left\|\Phi\left(g_{1}\right)-\Phi\left(g_{2}\right)\right\|_{L^{2}\left(\Gamma_{3}\right)}=\left\|R^{*}\left(\sigma_{\nu}\left(u_{g_{1}}\right)\right)-R^{*}\left(\sigma_{\nu}\left(u_{g_{2}}\right)\right)\right\|_{L^{2}\left(\Gamma_{3}\right)} .
$$

Using (2.14), the continuity of $R^{*}$ and (2.11), we deduce that there exists a constant $c>0$ such that

$$
\left\|\Phi\left(g_{1}\right)-\Phi\left(g_{2}\right)\right\|_{L^{2}\left(\Gamma_{3}\right)} \leq c\left\|u_{g_{1}}-u_{g_{2}}\right\|_{V} .
$$

Then

$$
\left\|\Phi\left(g_{1}\right)-\Phi\left(g_{2}\right)\right\|_{L^{2}\left(\Gamma_{3}\right)} \leq c C_{1}^{\prime}\|\mu\|_{L^{\infty}\left(\Gamma_{3}\right)}\left\|g_{1}-g_{2}\right\|_{L^{2}\left(\Gamma_{3}\right)} .
$$

If we set $\mu_{0}=1 / c C_{1}^{\prime}$, we conclude that for $\|\mu\|_{L^{\infty}\left(\Gamma_{3}\right)}<\mu_{0}$, the mapping $\Phi$ is a contraction and so it admits a unique fixed point $g_{*}$ and $\left(u_{g_{*}}, \beta_{g_{*}}\right)$ is a unique solution to Problem $Q_{n}^{i}$.

Lemma 3.7. There exist constants $C_{1}>0$ and $C_{2}>0$ such that

$$
\left\|u^{i+1}\right\|_{V} \leq C_{1}\left(\left\|f^{i+1}\right\|_{V}+1\right), \quad\left\|\Delta u^{i}\right\|_{V} \leq C_{2}\left(\left\|\Delta f^{i}\right\|_{V}+\Delta t\right)_{V} .
$$

Proof. By setting $v=0$ in the inequality of (3.1) we deduce

$$
a\left(u^{i+1}, u^{i+1}\right) \leq j\left(u^{i+1}, u^{i+1}\right)+r\left(\beta^{i+1}, u^{i+1}, u^{i+1}\right)+\left(f^{i+1}, u^{i+1}\right)_{V} .
$$

Using the properties of $j$ we have

$$
j\left(u^{i+1}, u^{i+1}\right) \leq\|\mu\|_{L^{\infty}\left(\Gamma_{3}\right)} d_{\Omega}\left\|R^{*}\left(\sigma_{\nu}\left(u^{i+1}\right)\right)\right\|_{L^{2}\left(\Gamma_{3}\right)}\left\|u^{i+1}\right\|_{V},
$$

and by (2.14), there exists a constant $C_{3}>0$ such that

$$
\left\|R^{*}\left(\sigma_{\nu}\left(u^{i+1}\right)\right)\right\|_{L^{2}\left(\Gamma_{3}\right)} \leq C_{3}\left(\left\|u^{i+1}\right\|_{V}+\left\|f^{i+1}\right\|_{V}\right) .
$$


On the other hand, using $\left|R\left(u_{\nu}\right)\right| \leq L,\left|R\left(u_{\tau}\right)\right| \leq L$ and (2.11), we have

$$
\left|r\left(\beta^{i+1}, u^{i+1}, u^{i+1}\right)\right| \leq d_{\Omega} L\left(\left\|c_{\nu}\right\|_{L^{\infty}\left(\Gamma_{3}\right)}+\left\|c_{\tau}\right\|_{L^{\infty}\left(\Gamma_{3}\right)}\right)\left\|u^{i+1}\right\|_{V} .
$$

Using (2.15)(b), we deduce the inequality

$$
\begin{gathered}
m\left\|u^{i+1}\right\|_{V}^{2} \leq\|\mu\|_{L^{\infty}\left(\Gamma_{3}\right)} d_{\Omega} C_{3}\left(\left\|u^{i+1}\right\|_{V}+\left\|f^{i+1}\right\|_{V}\right)\left\|u^{i+1}\right\|_{V}+\left\|f^{i+1}\right\|_{V}\left\|u^{i+1}\right\|_{V} \\
+d_{\Omega} L\left(\left\|c_{\nu}\right\|_{L^{\infty}\left(\Gamma_{3}\right)}+\left\|c_{\tau}\right\|_{L^{\infty}\left(\Gamma_{3}\right)}\right)\left\|u^{i+1}\right\|_{V},
\end{gathered}
$$

from which we infer that if

$$
\|\mu\|_{L^{\infty}\left(\Gamma_{3}\right)}<\frac{m}{d_{\Omega} C_{3}},
$$

then the first inequality of (3.6) holds for some constant $C_{1}>0$. To show the second inequality of (3.6) we consider the inequality of (3.1) translated at time $t_{i}$, that is,

$$
\begin{array}{r}
a\left(u^{i}, w-u^{i}\right)+j\left(u^{i}, w-u^{i-1}\right)-j\left(u^{i}, u^{i}-u^{i-1}\right)+r\left(\beta^{i}, u^{i}, u^{i+1}-u^{i}\right) \\
\geq\left(f^{i}, w-u^{i}\right)_{V} \quad \forall w \in V .
\end{array}
$$

Taking $w=u^{i}$ in the inequality of (3.1) and $w=u^{i+1}$ in the inequality (3.7) and adding the two inequalities, we obtain

$$
\begin{aligned}
& -a\left(\Delta u^{i}, \Delta u^{i}\right)-j\left(u^{i+1}, \Delta u^{i}\right)+j\left(u^{i}, u^{i+1}-u^{i-1}\right)-j\left(u^{i}, u^{i}-u^{i-1}\right) \\
& \quad+r\left(\beta^{i+1}, u^{i+1}, u^{i}-u^{i+1}\right)+r\left(\beta^{i}, u^{i}, u^{i+1}-u^{i}\right) \geq\left(-\Delta f^{i}, \Delta u^{i}\right)_{V} .
\end{aligned}
$$

Then using the inequality

$$
|| u_{\tau}^{i+1}-u_{\tau}^{i-1}|-| u_{\tau}^{i}-u_{\tau}^{i-1}|| \leq\left|u_{\tau}^{i+1}-u_{\tau}^{i}\right|,
$$

we have

$$
j\left(u^{i}, u^{i+1}-u^{i-1}\right)-j\left(u^{i}, u^{i}-u^{i-1}\right) \leq j\left(u^{i}, \Delta u^{i}\right) .
$$

Therefore

$$
\begin{gathered}
a\left(\Delta u^{i}, \Delta u^{i}\right) \leq j\left(u^{i}, \Delta u^{i}\right)-j\left(u^{i+1}, \Delta u^{i}\right)+r\left(\beta^{i+1}, u^{i+1},-\Delta u^{i}\right) \\
+r\left(\beta^{i}, u^{i}, \Delta u^{i}\right)+\left(\Delta f^{i}, \Delta u^{i}\right)_{V} .
\end{gathered}
$$

From (2.11), (2.14) and the continuity of $R^{*}$, it follows that exists a constant $c>0$ such that

$$
\left|-j\left(u^{i}, \Delta u^{i}\right)+j\left(u^{i+1}, \Delta u^{i}\right)\right| \leq c\|\mu\|_{L^{\infty}\left(\Gamma_{3}\right)}\left(\left\|\Delta u^{i}\right\|_{V}^{2}+\left\|\Delta f^{i}\right\|_{V}\left\|\Delta u^{i}\right\|_{V}\right) .
$$

Moreover, using, (2.11), $\left|R_{\nu}\left(u^{j}\right)\right| \leq L,\left|R_{\tau}\left(u^{j}\right)\right| \leq L, j=i, i+1$, and (3.4), we have

$$
\begin{aligned}
\mid r\left(\beta^{i+1}, u^{i+1},-\right. & \left.\Delta u^{i}\right)+r\left(\beta^{i}, u^{i}, \Delta u^{i}\right) \mid \\
& \leq L d_{\Omega}\left(\left\|c_{\nu}\right\|_{L^{\infty}\left(\Gamma_{3}\right)}+\left\|c_{\tau}\right\|_{L^{\infty}\left(\Gamma_{3}\right)}\right)\left\|\Delta u^{i}\right\|_{V}\left\|\Delta \beta^{i}\right\|_{L^{2}\left(\Gamma_{3}\right)} .
\end{aligned}
$$

As

$$
\left\|\Delta \beta^{i}\right\|_{L^{2}\left(\Gamma_{3}\right)} \leq \Delta t c_{1},
$$

where $c_{1}>0$, we combine the previous relations to deduce from inequality 
(3.8) that for

$$
c\|\mu\|_{L^{\infty}\left(\Gamma_{3}\right)}<m,
$$

there exists a constant $C_{2}>0$ such that the second inequality of (3.6) holds.

4. Existence of a solution. In this section we prove our main result, Theorem 2.1, which guarantees the existence of a weak solution for Problem $P_{2}$ obtained as a limit of discrete solutions. For this, we define the sequence of functions $u^{n}:[0, T] \rightarrow V$ by

$$
\begin{aligned}
u^{n}(t) & =u^{i}+\frac{t-t_{i}}{\Delta t} \Delta u^{i} \quad \text { on }\left[t_{i}, t_{i+1}\right], i=0, \ldots, n-1, \\
u^{n}(0) & =u_{0} .
\end{aligned}
$$

As in [6] we have the following lemma.

Lemma 4.1. There exists $u \in W^{1, \infty}(0, T ; V)$ and a subsequence of $\left(u^{n}\right)$, still denoted $\left(u^{n}\right)$, such that

$$
u^{n} \rightarrow u \quad \text { weak } k^{*} \text { in } W^{1, \infty}(0, T ; V) .
$$

Proof. Indeed, from (3.6) it follows that there exist constants $C_{3}>0$ and $C_{3}^{\prime}>0$ such that

$$
\left\|u^{n}\right\|_{W^{1, \infty}(0, T ; V)} \leq C_{3}\|f\|_{W^{1, \infty}(0, T ; V)}+C_{3}^{\prime} .
$$

Consequently, the sequence $\left(u^{n}\right)$ is bounded in $W^{1, \infty}(0, T ; V)$. Therefore, there exists a function $u \in W^{1, \infty}(0, T ; V)$ and a subsequence, still denoted by $\left(u^{n}\right)$, such that $u^{n} \rightarrow u$ weak $^{*}$ in $W^{1, \infty}(0, T ; V)$ as $n \rightarrow \infty$.

REMARK 4.2. As $W^{1, \infty}(0, T ; V) \hookrightarrow C([0, T] ; V)$ we have $u^{n}(t) \rightarrow u(t)$ weakly in $V$ for all $t \in[0, T]$.

Now we introduce the piecewise constant functions $\widetilde{u}^{n}:[0, T] \rightarrow V$ and $\widetilde{f}^{n}:[0, T] \rightarrow V$ defined as follows:

$$
\begin{aligned}
& \widetilde{u}^{n}(t)=u^{i+1}, \quad \widetilde{f}^{n}(t)=f\left(t_{i+1}\right), \quad \forall t \in\left(t_{i}, t_{i+1}\right], i=0, \ldots, n-1, \\
& \widetilde{u}^{n}(0)=u_{0}, \quad \widetilde{f}^{n}(0)=f(0) .
\end{aligned}
$$

As in [5] we have the following result.

Lemma 4.3. Passing to a subsequence again denoted $\left(\widetilde{u}^{n}\right)$ we have

(i) $\widetilde{u}^{n} \rightarrow u$ weak ${ }^{*}$ in $L^{\infty}(0, T ; V)$,

(ii) $\widetilde{u}^{n}(t) \rightarrow u(t)$ weakly in $V$ for a.e. $t \in[0, T]$,

(iii) $u(t) \in K$ for all $t \in[0, T]$.

Problem $P_{3}$. Find a bonding field $\beta:[0, T] \rightarrow L^{\infty}\left(\Gamma_{3}\right)$ such that

$$
\begin{aligned}
& \dot{\beta}(t)=-\left[\beta(t)\left(c_{\nu}\left(R_{\nu}\left(u_{\nu}(t)\right)\right)^{2}+c_{\tau}\left(\left|R_{\tau}\left(u_{\tau}(t)\right)\right|\right)^{2}\right)-\varepsilon_{a}\right]_{+} \quad \text { a.e. } t \in(0, T) \text {, } \\
& \beta(0)=\beta_{0} \quad \text { on } \Gamma_{3} \text {, }
\end{aligned}
$$

where $u$ is the weak solution found in Lemma 4.1. 
We have the following result.

Proposition 4.4. There exists a unique solution to Problem $P_{3}$ and it satisfies

$$
\beta \in W^{1, \infty}\left(0, T ; L^{2}\left(\Gamma_{3}\right)\right) \cap B .
$$

Proof. As in [5] let $k>0$ and let

$$
X=\left\{\beta \in C\left([0, T] ; L^{2}\left(\Gamma_{3}\right)\right): \sup _{t \in[0, T]}\left[\exp (-k t)\|\beta(t)\|_{L^{2}\left(\Gamma_{3}\right)}\right]<\infty\right\} .
$$

Then $X$ is a Banach space for the norm

$$
\|\beta\|_{X}=\sup _{t \in[0, T]}\left[\exp (-k t)\|\beta(t)\|_{L^{2}\left(\Gamma_{3}\right)}\right] .
$$

Consider the mapping $\Lambda: X \rightarrow X$ given by

$$
\Lambda \beta(t)=\beta_{0}-\int_{0}^{t}\left[\beta(s)\left(c_{\nu}\left(R_{\nu}\left(u_{\nu}(s)\right)\right)^{2}+c_{\tau}\left(\left|R_{\tau}\left(u_{\tau}(s)\right)\right|\right)^{2}\right)-\varepsilon_{a}\right]_{+} d s .
$$

Then there exists a constant $c_{1}^{\prime}>0$ such that

$$
\begin{aligned}
\mid \Lambda \beta_{1}(t)- & \left.\Lambda \beta_{2}(t)\right|^{2} \\
& \leq c_{1}^{\prime} \int_{0}^{t}\left(c_{\nu}\left(R_{\nu}\left(u_{\nu}(s)\right)\right)^{2}+c_{\tau}\left|R_{\tau}\left(u_{\tau}(s)\right)\right|^{2}\right)\left(\beta_{1}(t)-\beta_{2}(t)\right)^{2} d s .
\end{aligned}
$$

From $\left|R_{\nu}\left(u_{\nu}(s)\right)\right| \leq L$ and $\left|R_{\tau}\left(u_{\tau}(s)\right)\right| \leq L$, we deduce

$$
\begin{aligned}
\left\|\Lambda \beta_{1}(t)-\Lambda \beta_{2}(t)\right\|_{L^{2}\left(\Gamma_{3}\right)}^{2} & \leq c_{2}^{\prime} \int_{0}^{t}\left\|\beta_{1}(t)-\beta_{2}(t)\right\|_{L^{2}\left(\Gamma_{3}\right)}^{2} d s \\
& \leq c_{2}^{\prime}\left\|\beta_{1}-\beta_{2}\right\|_{X}^{2} \frac{\exp (2 k t)}{2 k} .
\end{aligned}
$$

It follows that

$$
\left\|\Lambda \beta_{1}-\Lambda \beta_{2}\right\|_{X} \leq \sqrt{\frac{c_{2}^{\prime}}{2 k}}\left\|\beta_{1}-\beta_{2}\right\|_{X}
$$

Therefore for $k$ sufficiently large, $\Lambda$ has a unique fixed point $\beta$. To prove that $\beta \in[0,1]$ for all $t \in[0, T]$, it suffices to invoke [20, Remark 3.1].

Now we introduce the sequences of functions $\left(\beta^{n}\right),\left(\widetilde{\beta}^{n}\right)$ defined on $[0, T]$ by

$$
\begin{aligned}
& \beta^{n}(t)=\beta^{i}+\frac{t-t_{i}}{\Delta t} \Delta \beta^{i} \quad \forall t \in\left[t_{i}, t_{i+1}\right], \\
& \widetilde{\beta}^{n}(t)=\beta^{i+1} \quad \forall t \in\left(t_{i}, t_{i+1}\right], i=0, \ldots, n-1, \\
& \widetilde{\beta}^{n}(0)=\beta^{n}(0)=\beta_{0} .
\end{aligned}
$$


We prove the following lemma.

LEMma 4.5. We have the following convergences

(i) $\beta^{n} \rightarrow \beta$ strongly in $L^{\infty}\left(0, T ; L^{2}\left(\Gamma_{3}\right)\right)$,

(ii) $\widetilde{\beta}^{n} \rightarrow \beta$ strongly in $L^{\infty}\left(0, T ; L^{2}\left(\Gamma_{3}\right)\right)$.

Proof. To show (i), since $\dot{\beta}^{n}(t)=\Delta \beta^{i} / \Delta t$, for all $t \in\left(t_{i}, t_{i+1}\right)$ we have

$$
\beta^{n}(t)=\beta^{i}-\int_{t_{i}}^{t}\left[\widetilde{\beta}^{n}(t)\left(c_{\nu}\left(R_{\nu}\left(\widetilde{u}_{\nu}^{n}(s)\right)\right)^{2}+c_{\tau}\left(\left|R_{\tau}\left(\widetilde{u}_{\tau}^{n}(s)\right)\right|\right)^{2}\right)-\varepsilon_{a}\right]_{+} d s,
$$

and also

$$
\beta(t)=\beta\left(t_{i}\right)-\int_{t_{i}}^{t}\left[\beta(t)\left(c_{\nu}\left(R_{\nu}\left(u_{\nu}(t)\right)\right)^{2}+c_{\tau}\left(\left|R_{\tau}\left(u_{\tau}(t)\right)\right|\right)^{2}\right)-\varepsilon_{a}\right]_{+} d s .
$$

Then

$$
\begin{aligned}
\beta^{n}(t)-\beta(t)= & \beta^{i}-\beta\left(t_{i}\right) \\
& -\int_{t_{i}}^{t}\left[\widetilde{\beta}^{n}(t)\left(c_{\nu}\left(R_{\nu}\left(\widetilde{u}_{\nu}^{n}(s)\right)\right)^{2}+c_{\tau}\left(\left|R_{\tau}\left(\widetilde{u}_{\tau}^{n}(s)\right)\right|\right)^{2}\right)-\varepsilon_{a}\right]_{+} d s \\
& +\int_{t_{i}}^{t}\left[\beta(t)\left(c_{\nu}\left(R_{\nu}\left(u_{\nu}(t)\right)\right)^{2}+c_{\tau}\left(\left|R_{\tau}\left(u_{\tau}(t)\right)\right|\right)^{2}\right)-\varepsilon_{a}\right]_{+} d s .
\end{aligned}
$$

Thus we have

$$
\begin{aligned}
\left\|\beta^{n}(t)-\beta(t)\right\|_{L^{2}\left(\Gamma_{3}\right)} \leq & \left\|\beta^{i}-\beta\left(t_{i}\right)\right\|_{L^{2}\left(\Gamma_{3}\right)} \\
& +\int_{0}^{t} \| \widetilde{\beta}^{n}(t)\left(c_{\nu}\left(R_{\nu}\left(\widetilde{u}_{\nu}^{n}(s)\right)\right)^{2}+c_{\tau}\left(\left|R_{\tau}\left(\widetilde{u}_{\tau}^{n}(s)\right)\right|\right)^{2}\right) \\
& -\beta(t)\left(c_{\nu}\left(R_{\nu}\left(u_{\nu}(t)\right)\right)^{2}+c_{\tau}\left(\left|R_{\tau}\left(u_{\tau}(t)\right)\right|\right)^{2}\right) \|_{L^{2}\left(\Gamma_{3}\right)} d s .
\end{aligned}
$$

Using the fact that $\left|R_{l}\left(u_{l}\right)\right| \leq L$ for $l=\nu, \tau$ (see [19]) and (3.4) we have

$$
\begin{aligned}
\left\|c_{\nu} \widetilde{\beta}^{n}(s)\left(R_{\nu}\left(\widetilde{u}_{\nu}^{n}(s)\right)\right)^{2}-c_{\nu} \beta(t)\left(R_{\nu}\left(u_{\nu}(s)\right)\right)^{2}\right\|_{L^{2}\left(\Gamma_{3}\right)} \\
\leq \| \widetilde{\beta}^{n}(s) c_{\nu}\left(\left(R_{\nu}\left(\widetilde{u}_{\nu}^{n}(s)\right)\right)^{2}-\left(R_{\nu}\left(u_{\nu}(s)\right)\right)^{2}\right) \\
\quad+\left(\widetilde{\beta}^{n}(s)-\beta^{n}(s)\right) c_{\nu}\left(R_{\nu}\left(u_{\nu}(s)\right)\right)^{2} \|_{L^{2}\left(\Gamma_{3}\right)} \\
\quad+\left\|\left(\beta^{n}(s)-\beta(s)\right) c_{\nu}\left(R_{\nu}\left(u_{\nu}(s)\right)\right)^{2}\right\|_{L^{2}\left(\Gamma_{3}\right)} \\
\leq 2 L\left\|c_{\nu}\right\|_{L^{\infty}\left(\Gamma_{3}\right)}\left\|\widetilde{u}_{\nu}^{n}(s)-u_{\nu}(s)\right\|_{L^{2}\left(\Gamma_{3}\right)} \\
\quad+L^{2}\left\|c_{\nu}\right\|_{L^{\infty}\left(\Gamma_{3}\right)}\left(\left\|\beta^{n}(s)-\beta(s)\right\|_{L^{2}\left(\Gamma_{3}\right)}+\Delta t c_{1}^{\prime}\right) .
\end{aligned}
$$


Also we have

$$
\begin{aligned}
\left\|\widetilde{\beta}^{n}(s) c_{\tau}\left(\left|R_{\tau}\left(\widetilde{u}_{\nu}^{n}(s)\right)\right|\right)^{2}-c_{\tau} \beta(t)\left(\left|R_{\tau}\left(u_{\tau}(t)\right)\right|\right)^{2}\right\|_{L^{2}\left(\Gamma_{3}\right)} \\
\leq \| \widetilde{\beta}^{n}(s) c_{\tau}\left(\left(\left|R_{\tau}\left(\widetilde{u}_{\tau}^{n}(s)\right)\right|\right)^{2}-\left(\left|R_{\tau}\left(u_{\tau}(s)\right)\right|\right)^{2}\right) \\
\quad+\left(\widetilde{\beta}^{n}(s)-\beta^{n}(s)\right) c_{\tau}\left(\left|R_{\tau}\left(u_{\tau}(s)\right)\right|\right)^{2} \|_{L^{2}\left(\Gamma_{3}\right)} \\
\quad+\left\|\left(\beta^{n}(s)-\beta(s)\right) c_{\tau}\left(\left|R_{\tau}\left(u_{\tau}(s)\right)\right|\right)^{2}\right\|_{L^{2}\left(\Gamma_{3}\right)} \\
\leq 2 L\left\|c_{\tau}\right\|_{L^{\infty}\left(\Gamma_{3}\right)}\left\|\widetilde{u}_{\tau}^{n}(s)-u_{\tau}(s)\right\|_{\left(L^{2}\left(\Gamma_{3}\right)\right)^{d}} \\
\quad+L^{2}\left\|c_{\tau}\right\|_{L^{\infty}\left(\Gamma_{3}\right)}\left(\left\|\beta^{n}(s)-\beta(s)\right\|_{L^{2}\left(\Gamma_{3}\right)}+\Delta t c_{1}^{\prime}\right) .
\end{aligned}
$$

From the previous inequalities we deduce

$$
\begin{aligned}
\left\|\beta^{n}(t)-\beta(t)\right\|_{L^{2}\left(\Gamma_{3}\right)} & \leq\left\|\beta^{i}-\beta\left(t_{i}\right)\right\|_{L^{2}\left(\Gamma_{3}\right)} \\
& +2 L\left(\left\|c_{\nu}\right\|_{L^{\infty}\left(\Gamma_{3}\right)} \int_{0}^{t}\left\|\widetilde{u}_{\nu}^{n}(s)-u_{\nu}(s)\right\|_{L^{2}\left(\Gamma_{3}\right)} d s\right. \\
& \left.+\left\|c_{\tau}\right\|_{L^{\infty}\left(\Gamma_{3}\right)} \int_{0}^{t}\left\|\widetilde{u}_{\tau}^{n}(s)-u_{\tau}(s)\right\|_{\left(L^{2}\left(\Gamma_{3}\right)\right)^{d}} d s\right) \\
& +L^{2}\left(\left\|c_{\nu}\right\|_{L^{\infty}\left(\Gamma_{3}\right)}+\left\|c_{\tau}\right\|_{L^{\infty}\left(\Gamma_{3}\right)}\right) \int_{0}^{t}\left\|\beta^{n}(s)-\beta(s)\right\|_{L^{2}\left(\Gamma_{3}\right)} d s \\
& +\left(\left\|c_{\nu}\right\|_{L^{\infty}\left(\Gamma_{3}\right)}+\left\|c_{\tau}\right\|_{L^{\infty}\left(\Gamma_{3}\right)}\right) T L^{2} \Delta t c_{1}^{\prime} .
\end{aligned}
$$

Now a Gronwall-type argument shows that there exists a constant $C_{4}>0$ such that

$$
\begin{aligned}
& \left\|\beta^{n}(t)-\beta(t)\right\|_{L^{2}\left(\Gamma_{3}\right)} \leq C_{4}\left(\left\|\beta^{i}-\beta\left(t_{i}\right)\right\|_{L^{2}\left(\Gamma_{3}\right)}\right. \\
& \left.\quad+\int_{0}^{t}\left(\left\|\widetilde{u}_{\nu}^{n}(s)-u_{\nu}(s)\right\|_{L^{2}\left(\Gamma_{3}\right)}+\left\|\widetilde{u}_{\tau}^{n}(s)-u_{\tau}(s)\right\|_{\left(L^{2}\left(\Gamma_{3}\right)\right)^{d}}\right) d s+\Delta t\right) .
\end{aligned}
$$

Hence

$$
\begin{aligned}
\max _{t \in[0, T]} & \left\|\beta^{n}(t)-\beta(t)\right\|_{L^{2}\left(\Gamma_{3}\right)} \leq C_{4}\left(\max _{i=0, \ldots, n}\left\|\beta^{i}-\beta\left(t_{i}\right)\right\|_{L^{2}\left(\Gamma_{3}\right)}\right. \\
& \left.+\int_{0}^{T}\left(\left\|\widetilde{u}_{\nu}^{n}(s)-u_{\nu}(s)\right\|_{L^{2}\left(\Gamma_{3}\right)}+\left\|\widetilde{u}_{\tau}^{n}(s)-u_{\tau}(s)\right\|_{\left(L^{2}\left(\Gamma_{3}\right)\right)^{d}}\right) d s+\Delta t\right) .
\end{aligned}
$$

As in [5, Lemma 3.5] we also have

$$
\lim _{n \rightarrow \infty} \max _{i=0, \ldots, n}\left\|\beta^{i}-\beta\left(t_{i}\right)\right\|_{L^{2}\left(\Gamma_{3}\right)}=0
$$

and from Lemma 4.3 we have $\widetilde{u}_{\nu}^{n}(s) \rightarrow u_{\nu}(s)$ strongly in $L^{2}\left(\Gamma_{3}\right)$, and 
$\widetilde{u}_{\tau}^{n}(s) \rightarrow u_{\tau}(s)$ strongly in $\left(L^{2}\left(\Gamma_{3}\right)\right)^{d}$ a.e. $s \in[0, T]$. Moreover, as

$$
\left\|\widetilde{u}_{\nu}^{n}(s)-u_{\nu}(s)\right\|_{L^{2}\left(\Gamma_{3}\right)} \leq d_{\Omega}\left\|\widetilde{u}^{n}(s)-u(s)\right\|_{V} \leq C^{\prime}\left(\|f\|_{W^{1, \infty}(0, T ; V)}+1\right),
$$

and

$$
\left\|\widetilde{u}_{\tau}^{n}(s)-u_{\tau}(s)\right\|_{\left(L^{2}\left(\Gamma_{3}\right)\right)^{d}} \leq d_{\Omega}\left\|\widetilde{u}^{n}(s)-u(s)\right\|_{V} \leq C^{\prime}\left(\|f\|_{W^{1, \infty}(0, T ; V)}+1\right),
$$

where $C^{\prime}>0$, it follows by using Lebesgue's theorem that

$$
\int_{0}^{T}\left(\left\|\widetilde{u}_{\nu}^{n}(s)-u_{\nu}(s)\right\|_{L^{2}\left(\Gamma_{3}\right)}+\left\|\widetilde{u}_{\tau}^{n}(s)-u_{\tau}(s)\right\|_{\left(L^{2}\left(\Gamma_{3}\right)\right)^{d}}\right) d s \rightarrow 0 \quad \text { as } n \rightarrow \infty .
$$

Hence we deduce

$$
\lim _{n \rightarrow \infty} \max _{t \in[0, T]}\left\|\beta^{n}(t)-\beta(t)\right\|_{L^{2}\left(\Gamma_{3}\right)}=0,
$$

and so (i) is proved. To prove (ii) it suffices to remark that there exists a constant $C_{5}>0$ such that

$$
\begin{aligned}
\left\|\widetilde{\beta}^{n}(t)-\beta(t)\right\|_{L^{2}\left(\Gamma_{3}\right)} & \leq\left\|\widetilde{\beta}^{n}(t)-\beta^{n}(t)\right\|_{L^{2}\left(\Gamma_{3}\right)}+\left\|\beta^{n}(t)-\beta(t)\right\|_{L^{2}\left(\Gamma_{3}\right)} \\
& \leq C_{5} \Delta t+\left\|\beta^{n}(t)-\beta(t)\right\|_{L^{2}\left(\Gamma_{3}\right)} .
\end{aligned}
$$

Now we have all the ingredients to prove the following proposition.

Proposition 4.6. $(u, \beta)$ is a solution to Problem $P_{2}$.

Proof. In the inequality of Problem $P_{n}^{i}$, for $v \in V$ set $w=u^{i}+v \Delta t$ and divide by $\Delta t$ to obtain

$$
\begin{aligned}
a\left(u^{i+1}, v-\frac{\Delta u^{i}}{\Delta t}\right)+ & j\left(u^{i+1}, v\right)-j\left(u^{i+1}, \frac{\Delta u^{i}}{\Delta t}\right)+r_{\tau}\left(\beta^{i+1}, u^{i+1}, v-\frac{\Delta u^{i}}{\Delta t}\right) \\
& \geq\left(f^{i+1}, v-\frac{\Delta u^{i}}{\Delta t}\right)_{V}+\left\langle\sigma_{\nu}\left(u^{i+1}\right), v_{\nu}-\frac{\Delta u_{\nu}^{i}}{\Delta t}\right\rangle_{\Gamma_{3}} .
\end{aligned}
$$

As in [5], we have

$$
\begin{aligned}
&\left\langle\sigma_{\nu}\left(u^{i+1}\right), v_{\nu}-\frac{\Delta u_{\nu}^{i}}{\Delta t}\right\rangle_{\Gamma_{3}}+r_{\nu}\left(\beta^{i+1}, u^{i+1}, v-\frac{\Delta u^{i}}{\Delta t}\right) \\
& \geq\left\langle\sigma_{\nu}\left(u^{i+1}\right), v_{\nu}\right\rangle_{\Gamma_{3}}+r_{\nu}\left(\beta^{i+1}, u^{i+1}, v\right) \quad \forall v \in V,
\end{aligned}
$$

and we deduce

$$
\begin{array}{r}
a\left(u^{i+1}, v-\frac{\Delta u^{i}}{\Delta t}\right)+j\left(u^{i+1}, v\right)-j\left(u^{i+1}, \frac{\Delta u^{i}}{\Delta t}\right)+r\left(\beta^{i+1}, u^{i+1}, v-\frac{\Delta u^{i}}{\Delta t}\right) \\
\geq\left(f^{i+1}, v-\frac{\Delta u^{i}}{\Delta t}\right)_{V}+\left\langle\sigma_{\nu}\left(u^{i+1}\right), v_{\nu}\right\rangle_{\Gamma_{3}}+r_{\nu}\left(\beta^{i+1}, u^{i+1}, v\right) \quad \forall v \in V .
\end{array}
$$


Hence for any $v \in L^{2}(0, T ; V)$, we have

$$
\begin{gathered}
a\left(\widetilde{u}^{n}(t), v(t)-\dot{u}^{n}(t)\right)+j\left(\widetilde{u}^{n}(t), v(t)\right)-j\left(\widetilde{u}^{n}(t), \dot{u}^{n}(t)\right) \\
+r\left(\widetilde{\beta}^{n}(t), \widetilde{u}^{n}(t), v(t)-\dot{u}^{n}(t)\right) \\
\geq\left(\widetilde{f}^{n}(t), v(t)-\dot{u}^{n}(t)\right)_{V}+\left\langle\sigma_{\nu}\left(\widetilde{u}^{n}(t)\right), v_{\nu}(t)\right\rangle_{\Gamma_{3}} \\
+r_{\nu}\left(\widetilde{\beta}^{n}(t), \widetilde{u}^{n}(t), v(t)\right) \quad \text { a.e. } t \in[0, T] .
\end{gathered}
$$

Integrating both sides on $(0, T)$, we obtain

$$
\begin{aligned}
\int_{0}^{T} a\left(\widetilde{u}^{n}(t), v(t)-\right. & \left.\dot{u}^{n}(t)\right) d t+\int_{0}^{T} j\left(\widetilde{u}^{n}(t), v(t)\right) d t-\int_{0}^{T} j\left(\widetilde{u}^{n}(t), \dot{u}^{n}(t)\right) d t \\
& +\int_{0}^{T} r\left(\widetilde{\beta}^{n}(t), \widetilde{u}^{n}(t), v(t)-\dot{u}^{n}(t)\right) d t \\
\geq & \int_{0}^{T}\left(\widetilde{f}^{n}(t), v(t)-\dot{u}^{n}(t)\right)_{V} d t+\int_{0}^{T}\left\langle\sigma_{\nu}\left(\widetilde{u}^{n}(t)\right), v_{\nu}(t)\right\rangle_{\Gamma_{3}} d t \\
& +\int_{0}^{T} r_{\nu}\left(\widetilde{\beta}^{n}(t), \widetilde{u}^{n}(t), v(t)\right) d t .
\end{aligned}
$$

Lemma 4.7. (i) For any $v \in L^{2}(0, T ; V)$ we have

$$
\begin{aligned}
\lim _{n \rightarrow \infty} \int_{0}^{T} a\left(\widetilde{u}^{n}(t), v(t)\right) d t & =\int_{0}^{T} a(u(t), v(t)) d t \\
\lim _{n \rightarrow \infty} \int_{0}^{T} j\left(\widetilde{u}^{n}(t), v(t)\right) d t & =\int_{0}^{T} j(u(t), v(t)) d t \\
\lim _{n \rightarrow \infty} \int_{0}^{T}\left(\widetilde{f}^{n}(t), v(t)-\dot{u}^{n}(t)\right)_{V} d t & =\int_{0}^{T}(f(t), v(t)-\dot{u}(t))_{V} d t, \\
\lim _{n \rightarrow \infty} \int_{0}^{T} r\left(\widetilde{\beta}^{n}(t), u^{n}(t), v(t)-\dot{u}^{n}(t)\right) d t & =\int_{0}^{T} r(\beta(t), u(t), v(t)-\dot{u}(t)) d t .
\end{aligned}
$$

(ii) We have

$$
\begin{aligned}
\liminf _{n \rightarrow \infty} \int_{0}^{T} a\left(\widetilde{u}^{n}(t), \dot{u}^{n}(t)\right) d t & \geq \int_{0}^{T} a(u(t), \dot{u}(t)) d t, \\
\liminf _{n \rightarrow \infty} \int_{0}^{T} j\left(\widetilde{u}^{n}(t), \dot{u}^{n}(t)\right) d t & \geq \int_{0}^{T} j(u(t), \dot{u}(t)) d t .
\end{aligned}
$$

Proof. For the proof of (4.2)-(4.4) and (4.6)-(4.7) we refer the reader to [6, Lemmas 4-6]. To prove (4.5) it suffices to invoke [5, Lemma 3.8] and use the properties (3.4). 
Now using Lemmas 4.5 (ii) and 4.7 we let $n \rightarrow \infty$ in (4.1) to obtain

$$
\begin{gathered}
\int_{0}^{T} a(u(t), v(t)-\dot{u}(t)) d t+\int_{0}^{T} j(u(t), v(t)) d t-\int_{0}^{T} j(u(t), \dot{u}(t)) d t \\
\quad+\int_{0}^{T} r(\beta(t), u(t), v(t)-\dot{u}(t)) d t \\
\geq \int_{0}^{T}(f(t), v(t)-\dot{u}(t))_{V} d t+\int_{0}^{T}\left\langle\sigma_{\nu}(u(t)), v_{\nu}(t)\right\rangle_{\Gamma_{3}} d t \\
\quad+\int_{0}^{T} r_{\nu}(\beta(t), u(t), v(t)) d t .
\end{gathered}
$$

On the other hand, from the inequality in (3.1) we deduce

$$
\begin{aligned}
a\left(u^{i+1}, w-u^{i+1}\right)+j\left(u^{i+1}, w-u^{i+1}\right) & +r\left(\beta^{i+1}, u^{i+1}, w-u^{i+1}\right) \\
& \geq\left(f^{i+1}, w-u^{i+1}\right)_{V} \quad \forall w \in K,
\end{aligned}
$$

and so for all $t \in(0, T]$,

$$
\begin{aligned}
a\left(\widetilde{u}^{n}(t), w-\widetilde{u}^{n}(t)\right)+j\left(\widetilde{u}^{n}(t), w-\widetilde{u}^{n}(t)\right)+ & r\left(\widetilde{\beta}^{n}(t), \widetilde{u}^{n}(t), w-\widetilde{u}^{n}(t)\right) \\
& \geq\left(\widetilde{f}^{n}, w-\widetilde{u}^{n}(t)\right)_{V} \quad \forall w \in K .
\end{aligned}
$$

Passing to the limit we obtain, for all $t \in[0, T]$,

$$
\begin{aligned}
a(u(t), w-u(t))+j(u(t), w-u(t))+r(\beta(t), u(t), w-u(t)) & \\
& \geq(f, w-u(t))_{V} \quad \forall w \in K .
\end{aligned}
$$

This inequality implies, by applying Green's formula, that for all $t \in[0, T]$,

$$
\left\langle\sigma_{\nu}(u(t)), v_{\nu}-u_{\nu}(t)\right\rangle_{\Gamma_{3}}+r_{\nu}(\beta(t), u(t), v-u(t)) \geq 0 \quad \forall v \in K,
$$

and so we get (2.21), which implies

$$
\left\langle\sigma_{\nu}(u(t)), \dot{u}_{\nu}(t)\right\rangle_{\Gamma_{3}}+r_{\nu}(\beta(t), u(t), \dot{u}(t))=0 .
$$

Combining (4.8) and (4.9) we obtain

$$
\begin{aligned}
& \int_{0}^{T} a(u(t), v(t)-\dot{u}(t)) d t+\int_{0}^{T} j(u(t), v(t)) d t-\int_{0}^{T} j(u(t), \dot{u}(t)) d t \\
& \quad+\int_{0}^{T} r(\beta(t), u(t), v(t)-\dot{u}(t)) d t \\
& \geq \int_{0}^{T}(f(t), v(t)-\dot{u}(t))_{V} d t+\int_{0}^{T}\left\langle\sigma_{\nu}(u(t)), v_{\nu}(t)-\dot{u}_{\nu}(t)\right\rangle_{\Gamma_{3}} d t \\
& \quad+\int_{0}^{T} r_{\nu}(\beta(t), u(t), v(t)-\dot{u}(t)) d t .
\end{aligned}
$$

As in [5] from the inequality (4.10) we deduce (2.20). 
Conclusion. In this paper we have studied a mathematical model which describes a quasistatic frictional contact problem with adhesion for elastic materials. The adhesive and frictional contact is modelled with the Signorini condition and the non-local Coulomb friction law. We have proved existence of a weak solution under a smallness assumption on the friction coefficient. The important question of uniqueness still remains open.

\section{References}

[1] H. Brezis, Équations et inéquations non linéaires dans les espaces vectoriels en dualité, Ann. Inst. Fourier (Grenoble) 18 (1968), 115-175.

[2] L. Cangémi, Frottement et adhérence : modèle, traitement numérique et application à l'interface fibre/matrice, Ph.D. Thesis, Univ. Méditerranée, Aix Marseille I, 1997.

[3] O. Chau, J. R. Fernandez, M. Shillor and M. Sofonea, Variational and numerical analysis of a quasistatic viscoelastic contact problem with adhesion, J. Comput. Appl. Math. 159 (2003), 431-465.

[4] O. Chau, M. Shillor and M. Sofonea, Dynamic frictionless contact with adhesion, J. Appl. Math. Phys. (ZAMP) 55 (2004), 32-47.

[5] M. Cocu and R. Rocca, Existence results for unilateral quasistatic contact problems with friction and adhesion, Math. Model. Numer. Anal. 34 (2000), 981-1001.

[6] M. Cocu, E. Pratt and M. Raous, Formulation and approximation of quasistatic frictional contact, Int. J. Engrg. Sci. 34 (1996), 783-798.

[7] G. Duvaut, Équilibre d'un solide élastique avec contact unilatéral et frottement de Coulomb, C. R. Acad. Sci. Paris Sér. A 290 (1980), 263-265.

[8] G. Duvaut et J.-L. Lions, Les inéquations en mécanique et en physique, Dunod, Paris, 1972.

[9] J. R. Fernández, M. Shillor and M. Sofonea, Analysis and numerical simulations of a dynamic contact problem with adhesion, Math. Comput. Modelling 37 (2003), $1317-1333$.

[10] M. Frémond, Adhérence des solides, J. Mécanique Théor. Appl. 6 (1987), 383-407.

[11] —, Équilibre des structures qui adhèrent à leur support, C. R. Acad. Sci. Paris Sér. II 295 (1982), 913-916.

[12] -, Non Smooth Thermomechanics, Springer, Berlin, 2002.

[13] S. A. Nassar, T. Andrews, S. Kruk and M. Shillor, Modelling and simulations of a bonded rod, Math. Comput. Modelling 42 (2005), 553-572.

[14] M. Raous, L. Cangémi and M. Cocu, A consistent model coupling adhesion, friction, and unilateral contact, Comput. Methods Appl. Mech. Engrg. 177 (1999), 383-399.

[15] M. Raous, M. Jean and J. J. Moreau (eds.), Contact Mechanics, Plenum Press, New York, 1995.

[16] J. Rojek and J. J. Telega, Contact problems with friction, adhesion and wear in orthopeadic biomechanics. I, General developments, J. Theor. Appl. Mech. 39 (2001), 655-677.

[17] M. Shillor, M. Sofonea and J. J. Telega, Models and Variational Analysis of Quasistatic Contact, Lecture Notes in Phys. 655, Springer, Berlin, 2004.

[18] M. Sofonea, R. Arhab and R. Tarraf, Analysis of electroelastic frictionless contact problems with adhesion, J. Appl. Math. 2006, Art. ID 64217, 25 pp. 
[19] M. Sofonea, W. Han and M. Shillor, Analysis and Approximations of Contact Problems with Adhesion or Damage, Pure Appl. Math. 276, Chapman \& Hall/CRC Press, Boca Raton, FL, 2006.

[20] M. Sofonea and T. V. Hoarau-Mantel, Elastic frictionless contact problems with adhesion, Adv. Math. Sci. Appl. 15 (2005), 49-68.

Laboratoire de Systèmes Dynamiques

Faculté de Mathématiques, USTHB

BP 32 El Alia

Bab Ezzouar, 16111, Algeria

E-mail: atouzaline@yahoo.fr 
